\title{
An Improved Method for Generating Cutter Points Based on Required Form Accuracy of the Machining Surface
}

\author{
Shijun Ji, Huijuan Yu, and Ji Zhao \\ School of Mechanical Science and Engineering, Jilin University, Changchun 130025, China \\ Correspondence should be addressed to Ji Zhao; jzhao@jlu.edu.cn
}

Received 21 October 2014; Revised 24 March 2015; Accepted 30 March 2015

Academic Editor: María I. Herreros

Copyright ( 2015 Shijun Ji et al. This is an open access article distributed under the Creative Commons Attribution License, which permits unrestricted use, distribution, and reproduction in any medium, provided the original work is properly cited.

\begin{abstract}
Recently, freeform surfaces are widely used in optical field. Fabrication of freeform surface optics needs high machining accuracy and high efficiency simultaneously. Using the present ultraprecision manufacturing technologies, the form accuracy of freeform surfaces can reach submicrometric or even nanometer range but at the cost of low efficiency; moreover, the form accuracy cannot be predicted; the number and the position of cutter points cannot be accurately controlled before practical machining. A novel strategy of cutter points distribution is proposed in the paper, which is based on the required accuracy of the freeform surface and the machining efficiency can be improved by eliminating redundant cutter points on the processing trajectory. The new idea is detailed, simulations are conducted, and experiments are done to verify the feasibility of the proposed method in fabricating two sinusoidal ring surfaces.
\end{abstract}

\section{Introduction}

With the development of high-technology, the optical freeform components have become more and more widely applied in recent years, especially in advanced optical system. For such system, the freeform components can minimize system sizes, lower weight, enhance imaging quality, and provide some other advantages [1-3]. Using the current micro- and ultraprecision machining techniques, the optical freeform surface can be achieved to a few nanometers in roughness and submicron in form error $[4,5]$, while the high quality generally is at the cost of lower efficiency and the form accuracy cannot be predicted ahead of actual machining. To meet the growing demands on high-precision quality with high efficiency, the study of this issue becomes more and more essential.

There are many literatures analyzing the efficiency issue from different topics, such as optimizing machining parameters [6-8], modelling, and simulation of surface roughness [9, 10]. However, few papers concentrate on the optimization of cutter point distribution. According to traditional methods, the more the number of cutter points are, the higher the quality of machined surface will be. While this strategy ignores the case that some cutter points are not necessary to obtain high-precision surface, on the contrary, they may decrease the machining efficiency, so the effective cutter points are very important for the improvement of machining efficiency.

For cutter point, the commonly used generation methods are getting the point equally spaced angles and equally spaced chords in plane spiral tool path [11, 12]. Although the cutter points generated by these ways can both meet the precision acquirements through multiple-stage processes, one disadvantage of these methods is that the form accuracy cannot be controlled before actual machining and the form accuracy only can be known after measurement. Besides, the obtained form accuracy is uniform on the whole machined surface, which is related to the profile of surface to be machined.

In this paper, the cutter point generation method based on form accuracy control in whole surface is studied, and the single point diamond turning (SPDT) technology is adopted to analyze the improved method. The main purpose is to generate the effective cutter point according to the given form accuracy requirement, which can both increase the machining efficiency and predict the precision ahead 


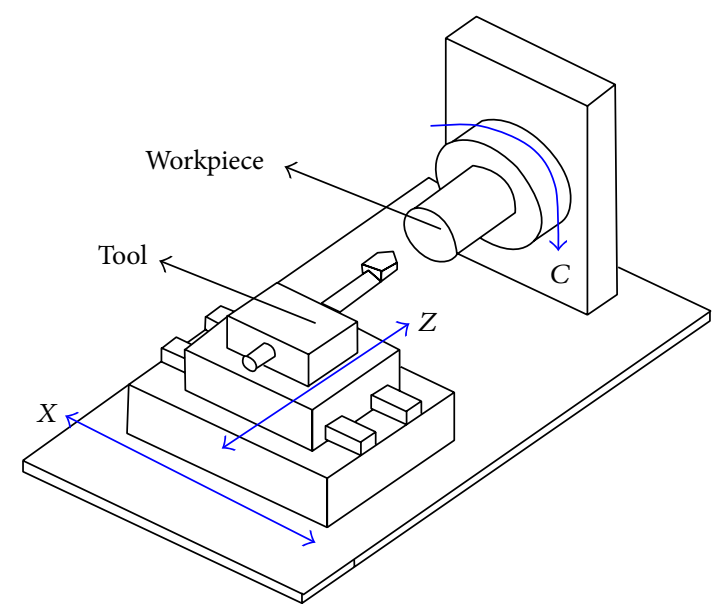

FIGURE 1: The model of the ultraprecision diamond turning machining tool.

of machining. To verify the novel cutter points' generation method, experiments are done for machining two sinusoidal ring surfaces with different required form accuracy.

\section{Cutter Point of Equally Spaced Angles}

For SPDT, the trajectory of diamond tool is composed by a series of cutter points. Generally, the model of the ultraprecision diamond turning machining tool is shown in Figure 1, and the cutter points are firstly generated in $X Z$ plane spiral, and the $Z$-value of points can be calculated according to the corresponding radial position and spindle rotation angle. Then, the three-dimensional coordinate of cutter points can be confirmed.

Equally spaced angles is the most common method used to generate cutter points on $X Z$-plane spiral cutter tool path, and some typical complex surfaces with high precision are machined using the cutter points generated by equally spaced angles, such as off-axis aspheric surface $[1,3,13]$ and microlens array [14-16]. Equally spaced angles refering to the central angles are equal for any adjacent cutter points on the same spiral revolution. The schematic diagram of equally spaced angles is shown in Figure 2.

The form accuracy of machined surface can be seen as a measure which is used to evaluate the effective of the adopted cutter points. Since the ideal curve is approximated by polylines which composed by cutter points, the chord error must be existent between ideal curve and approximated polylines. So, the form accuracy can be reflected by chord error of cutter tool path, and the smaller chord error is, the higher form accuracy is.

To further analyze the characteristic of equally spaced angle points, the sinusoidal ring surface is taken as the example. The equation of sinusoidal ring surface is presented as

$$
\begin{aligned}
z & =A \sin \left(w \sqrt{x^{2}+y^{2}}\right)+k, \\
x^{2}+y^{2} & \leq R^{2},
\end{aligned}
$$

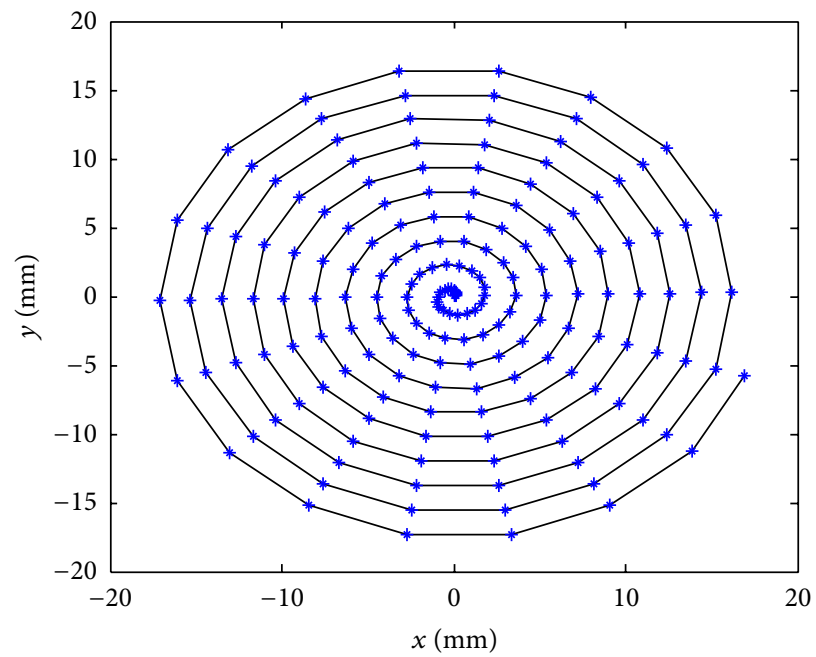

FIGURE 2: Schematic diagram of equally spaced angles.

where $A$ is the amplitude, $w$ is the angular velocity, and $k$ is the offset distance of the sinusoidal surface. $R$ is the radius of cylindrical workpiece. Let $A=0.2 \mathrm{~mm}, w=\pi, k=$ $-30 \mathrm{~mm}$, and $R=18 \mathrm{~mm}$; the cutter points generated with equally spaced angles on $X Z$-plane is displayed in Figure 2. The practical cutter tool path is three-dimensional and the chord error of space cutter tool path can transform into that of relation curve between radial position $r$ and $z$-coordinate. The cutter point generated using equally spaced angles in $r-z$ curve is illustrated in Figure 3, where the chord error is convenient to be analyzed and calculated. According to the used plane spiral, the total number of generated cutter points is 255 . The change of chord error for two adjacent points in $r-z$ curve is demonstrated in Figure 4. As shown in Figure 4, the chord error has great fluctuation and the trend of variation is similar to sinusoid. The chord error has the range from about $2.42 \mu \mathrm{m}$ to $0.304 \mu \mathrm{m}$, which cannot reach the requirement of the harmoniousness for form accuracy in whole surface. 


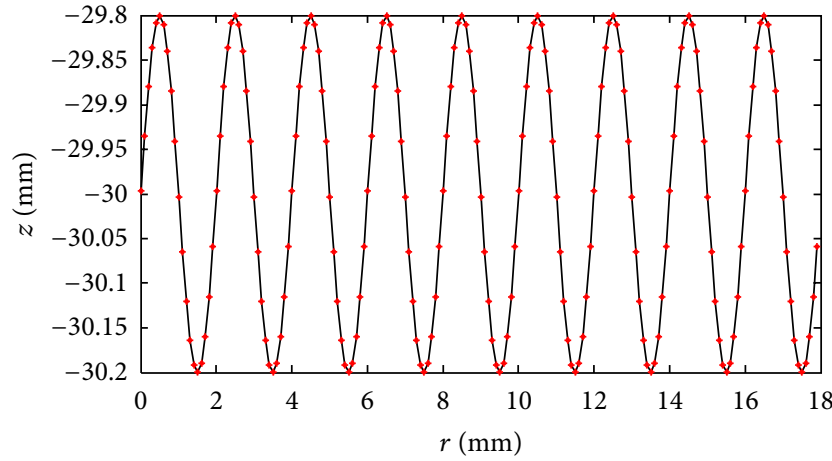

FIGURE 3: The cutter points of equally spaced angles in $r$ - $z$ curve.

It can be found that the equally spaced angles cannot achieve the prediction of form accuracy when generating the cutter points, which not only lower the efficiency, but also lower the quality of whole surface, so it is very necessary to research a method that generates the cutter point according to the limitation of form accuracy in whole surface.

\section{Method of Form Accuracy Control}

The main idea of form accuracy control proposed in this paper is generating cutter point based on the given requirement for form accuracy rather than confirmation about the range of accuracy after generating the point. This new method can predict form accuracy and increase the harmoniousness of accuracy in whole surface.

The schematic diagram of the proposed method is indicated in Figure 5, where the spiral tool path is converted into the curve $z=f(r)$. Suppose $P_{i}\left(r_{i}, z_{i}\right)$ is the original point and the chord error $h$ is to be controlled. Assume that $P_{i+1}\left(r_{i+1}, z_{i+1}\right)$ is the next point to be solved and $P_{0}\left(r_{0}, z_{0}\right)$ is the location where $h$ is located, respectively. The chord error $h$ can be expressed as

$h$

$$
=\frac{\left|A w r_{0} \cos \left(w r_{0}\right)-A \sin \left(w r_{0}\right)+A \sin \left(w r_{i}\right)-A w r_{i} \cos \left(w r_{0}\right)\right|}{\sqrt{1+\left(A w \cos \left(w r_{i+1}\right)\right)^{2}}} .
$$

Notice that the slope of curve in $P_{0}\left(r_{0}, z_{0}\right)$ is equal to the slope of straight line $P_{i} P_{i+1}$; the equation expressing the slope is

$$
A w \cos \left(w r_{0}\right)=\frac{A \sin \left(w r_{i+1}\right)-A \sin \left(w r_{i}\right)}{r_{i+1}-r_{i}},
$$

so, according to $P_{i}$ and $h$, the $r_{i+1}$ of $P_{i+1}$ can be calculated from (2) and (3). The $z_{i+1}$ of $P_{i+1}$ can be computed by curve equation $z=f(x)$, and the point $P_{i+1}\left(x_{i+1}, z_{i+1}\right)$ can be solved. Similarly, taking $P_{i+1}\left(x_{i+1}, z_{i+1}\right)$ as the original point, the next point also can be obtained using the above solution process. Consequently, all cutter points on tool path can be generated in this way.

To verify the effectiveness of the proposed cutter point generation method, cutter points of sinusoidal ring surface are generated under the condition of two kinds of form

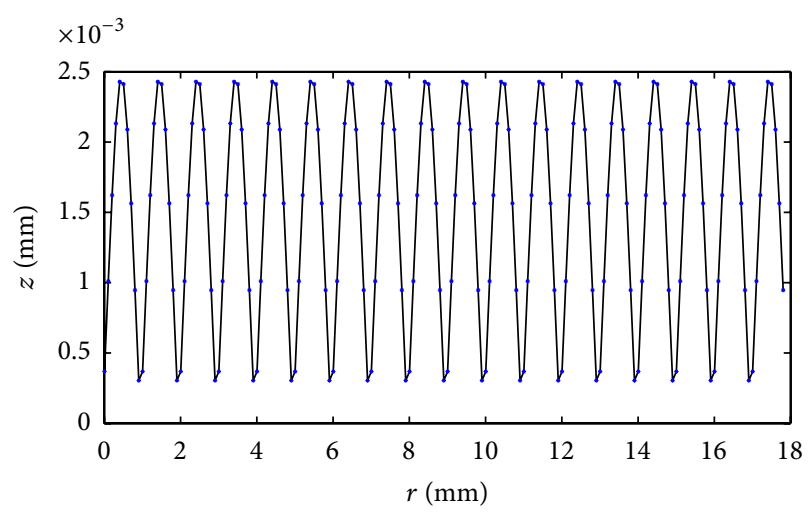

FIGURE 4: The change of chord error for two adjacent points of equally spaced angles in $r-z$ curve.

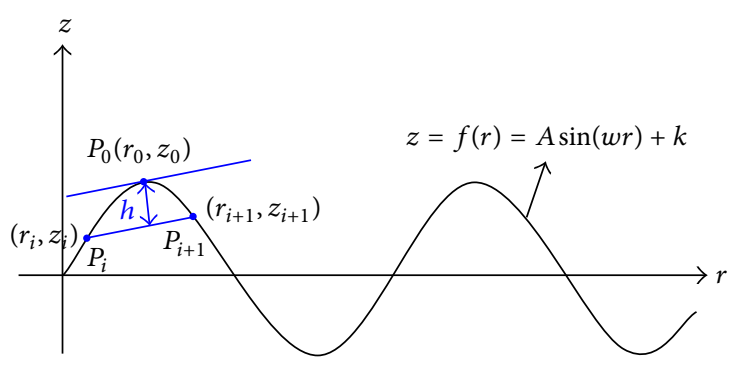

FIGURE 5: The schematic diagram of proposed method for cutter point generation.

accuracy, which are $1 \mu \mathrm{m}$ and $0.1 \mu \mathrm{m}$, respectively. Let the location of initial point be $(0,-30)$, and the cutter point can be obtained by (2). The generated two kinds of cutter points on tool path are displayed in Figure 6. As shown in Figures $6(\mathrm{a})$ and $6(\mathrm{~b})$, the number of effective cutter points is 200 and 650 when the chord error requires $1 \mu \mathrm{m}$ and $0.1 \mu \mathrm{m}$, respectively. Compared with the number of cutter points and the form accuracy for traditional equally spaced angles method mentioned in Figures 3 and 4, the machining effectiveness and form accuracy in whole surface are greatly improved for the proposed cutter point generation method.

To test the practicability of the proposed method, the chord error for produced tool path composed of calculated cutter points is computed, and the result is demonstrated in Figure 7. Figures 7(a) and 7(b) show that the chord errors are basically kept in $1 \mu \mathrm{m}$ and $0.1 \mu \mathrm{m}$, respectively, although the error is oscillated in range of the required accuracy. Figure 7 demonstrates that the improved cutter point generation method predicts the form accuracy and controls the form accuracy in whole surface before actual machining.

\section{Experiment and Analysis}

Based on the above theory, two sinusoidal ring surfaces with the corresponding parameters given above were fabricated in ultraprecision machining system, the Nanoform250. Material of the workpieces is Al-6061. A diamond tool with the nose 


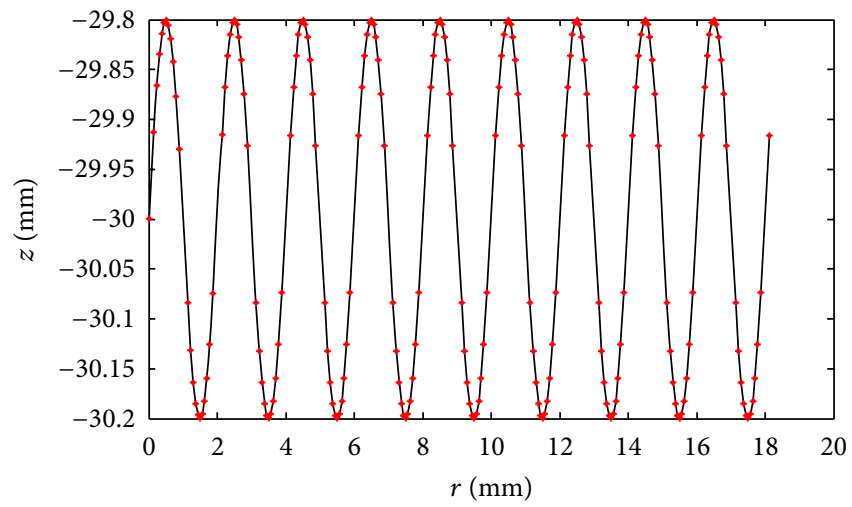

(a)

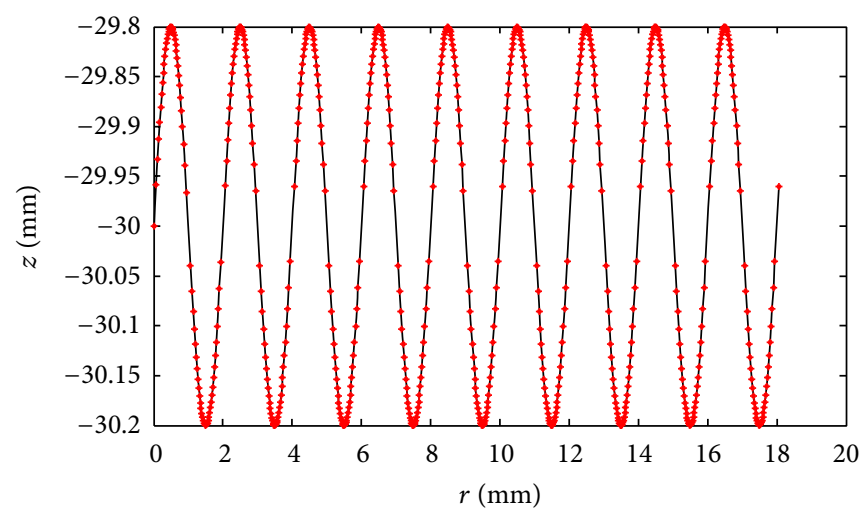

(b)

FIGURE 6: Two kinds of generated cutter points with proposed method (a) chord error is $1 \mu \mathrm{m}$ (b) chord error is $0.1 \mu \mathrm{m}$.

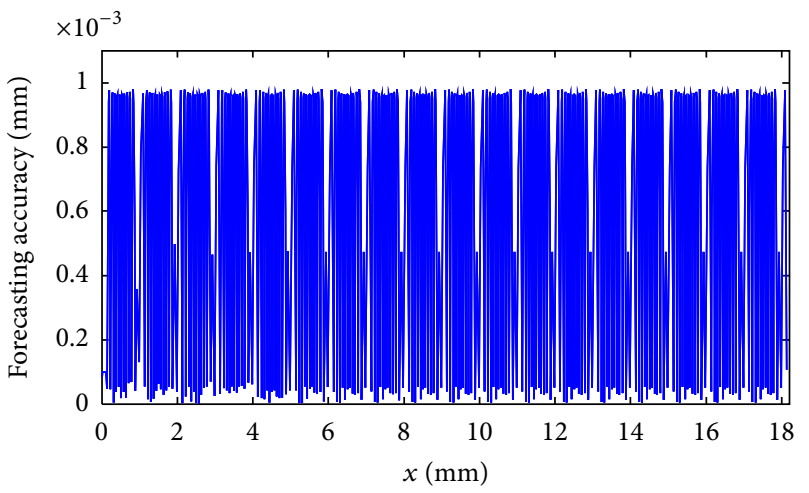

(a)

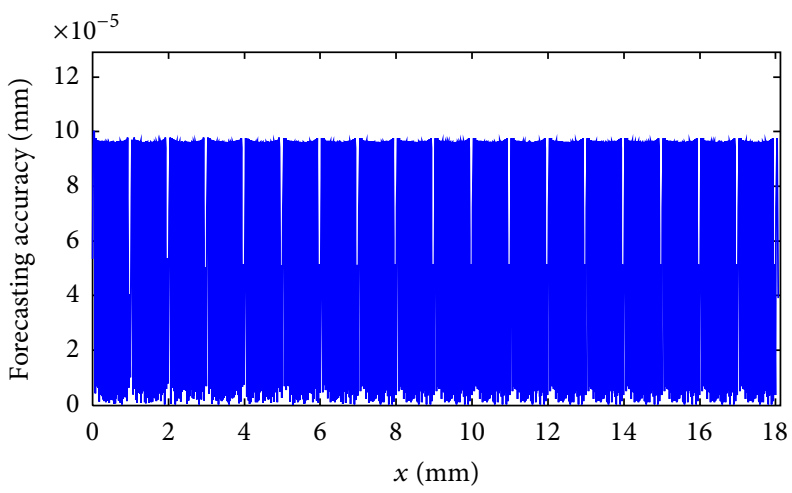

(b)

FIGURE 7: The error between theoretical curve and polyline constituted of cutter points with proposed method; (a) the predefined chord error is $1 \mu \mathrm{m}$ (b) the predefined chord error is $0.1 \mu \mathrm{m}$.

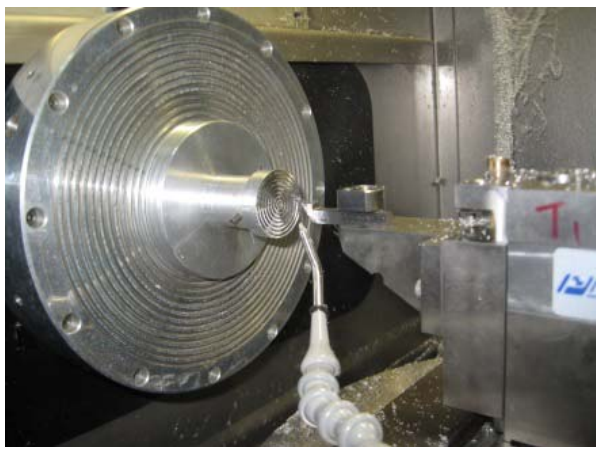

(a)

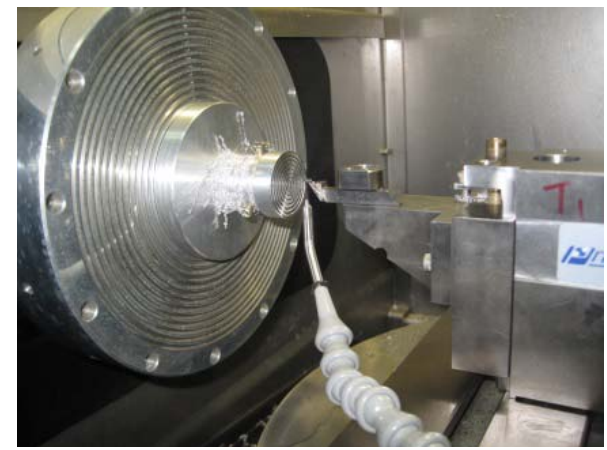

(b)

FIGURE 8: Machining process (a) chord error is $1 \mu \mathrm{m}$ (b) chord error is $0.1 \mu \mathrm{m}$.

radius of $0.506 \mathrm{~mm}$, including angle of $120^{\circ}$ and clearance angle of $10^{\circ}$, was applied. Figure 8 gives the real machining situation. In the cutting process, the spindle speed is set to $2500 \mathrm{rpm}$, the cutting depth is set to be $10 \mu \mathrm{m}$. The form accuracy of the machined surfaces is measured by the TAYLOR
HOBSON, which is a high-precision measuring instrument. The measurement processes are displayed in Figure 9. The measured curves of $1 \mu \mathrm{m}$ predefined sinusoidal ring surface and $0.1 \mu \mathrm{m}$ predefined sinusoidal ring surface are given by Figures 10 and 11, respectively. 


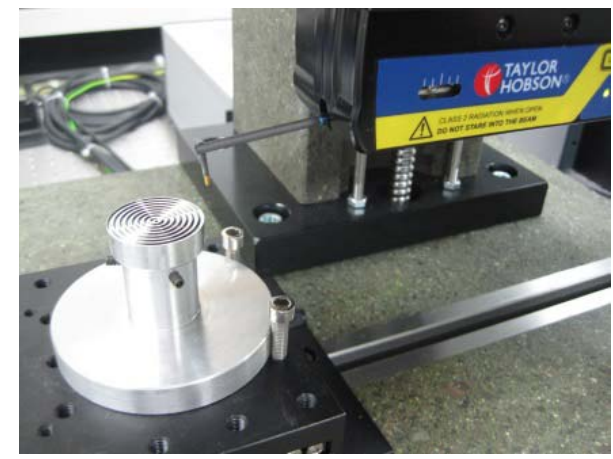

(a)

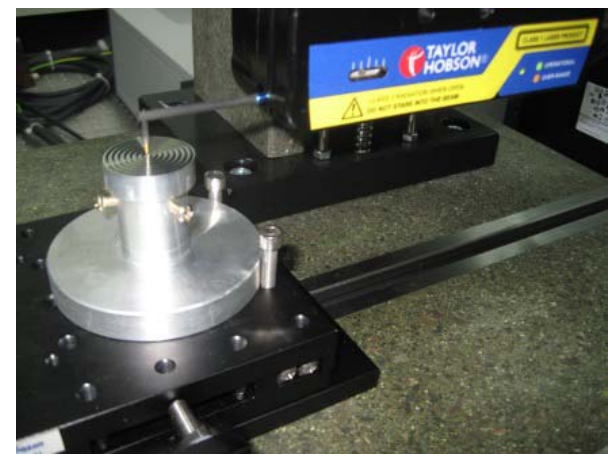

(b)

FIGURE 9: Diagram of measurement (a) chord error is $1 \mu \mathrm{m}$ (b) chord error is $0.1 \mu \mathrm{m}$.

Figure 10(a) gives the original measured curve of the machined sinusoidal ring surface with the $1 \mu \mathrm{m}$ predefined form accuracy. Figure 10(b) displays the matching graph about the theoretical curve and the corresponding practical measurement curve which is obtained by rotating and translating the original curve in the analysis area of Figure 10(a). To analyze the deviation between the theoretical curve and the measured curve, it is necessary to determine where the maximum deviation exits. In fact, the maximum deviations are located at the regions with the maximum curvature of the curve, therefore, the maximum deviations of the two curves is located at peek and trough of the sinusoidal curve. Therefore, the peek area and trough area of the sinusoidal curve are chosen to study deviation, as shown by regions marked "Region A" and "Region C" in Figure 10(b) or displayed by Figures $10(\mathrm{c})$ and $10(\mathrm{e})$. As a supplementary, the middle region between a peek area and its nearest trough area is also chosen to study the deviation, as shown by regions marked "Region B" in Figure 10(b) or displayed by Figure 10(d). From Figures $10(\mathrm{c})$ and $10(\mathrm{e})$, it can be seen that the maximum deviations located at peek and trough are about $2 \mu \mathrm{m}$ and $1 \mu \mathrm{m}$, respectively. From Figure 10(d), it can be seen that the maximum deviation of the middle region between peek and trough is about $1 \mu \mathrm{m}$.

Figure 11(a) gives the original measured curve of the machined sinusoidal ring surface with the $0.1 \mu \mathrm{m}$ predefined form accuracy. Figure 11(b) displays the matching graph about the theoretical curve and the corresponding practical measurement curve which is obtained by rotating and translating the original curve in the analysis area of Figure 11(a). To analyze the deviation between the theoretical curve and the measured curve, the peek area and trough area of the sinusoidal curve are chosen to study deviation, as shown by regions marked "Region D" and "Region F" in Figure 11(b) or displayed by Figures 11(c) and 11(e). As a supplementary, the middle region between a peek area and its nearest trough area is also chosen to study the deviation, as shown by regions marked "Region E" in Figure 11(b) or displayed by Figure 11(d). From Figures 11(c) and 11(e), it can be seen the the maximum deviations located at peek and trough are about $0.3 \mu \mathrm{m}$ and $0.2 \mu \mathrm{m}$. From Figure $11(\mathrm{~d})$, it can be seen the maximum deviation of the middle region between peek and trough is about $0.1 \mu \mathrm{m}$.

In real machining process, the PVT interpolating mode is used. Usually, execution time of each instruction is a constant, then total executing time of the program for predefined $0.1 \mu \mathrm{m}$ form accuracy surface is about $3.25(650 / 200)$ times as that for the predefined $1 \mu \mathrm{m}$ form accuracy surface, whereas the form accuracy of the $0.1 \mu \mathrm{m}$ form accuracy surface is about 10 times as that for the predefined $1 \mu \mathrm{m}$ form accuracy surface.

\section{Conclusions}

Based on the theoretical analysis and experimental verification, the following conclusions can be drawn.

(1) A novel cutter point generation method is presented based on the required form accuracy of the machining surface; this method can eliminate redundant cutter points, improve the machining efficiency, and predict the number and locations of the cutter points.

(2) Simulations are conducted for two sinusoidal ring surfaces with $0.1 \mu \mathrm{m}$ and $1 \mu \mathrm{m}$ form accuracy, respectively, the deviation of the whole surface is predicted, and the number and locations of the cutter points are determined in advance.

(3) Two Al-6061 workpieces with sinusoidal ring surfaces are machined which are based on the cutter points generated by the novel method. The profiles of the two machined sinusoidal ring surfaces are measured and analyzed. It can be found that, under different required form accuracy, different practical machined surfaces will be obtained. The practicability of the proposed method can be verified. 


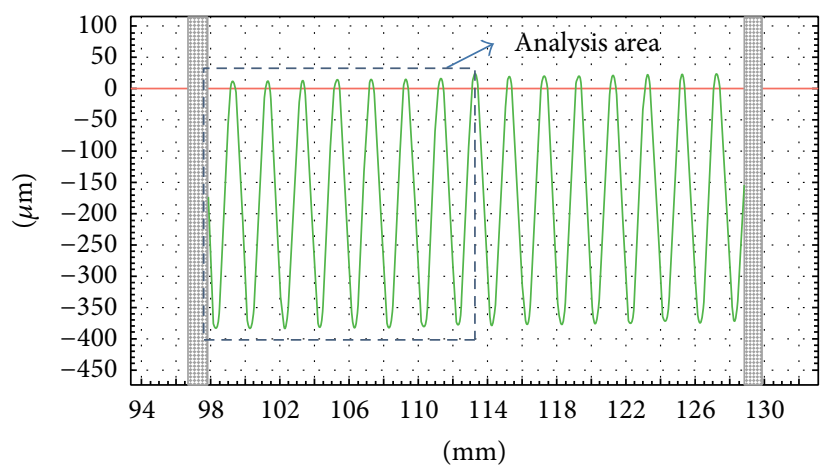

(a)

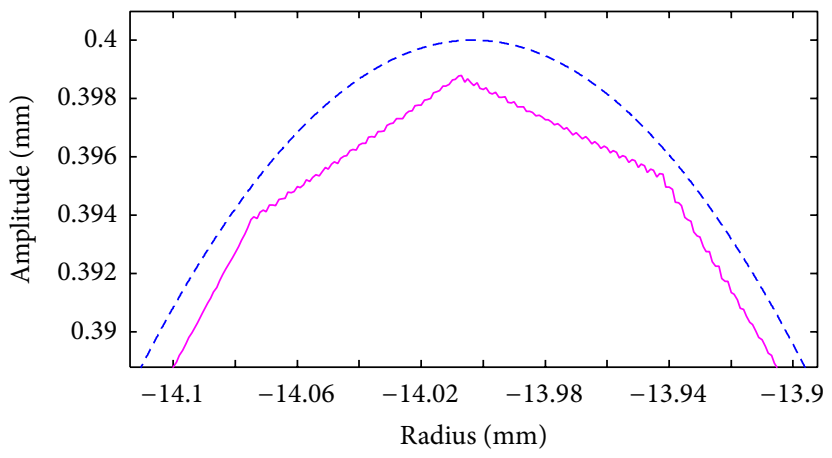

_ Measuring curve
- - Theoretical curve

(c)

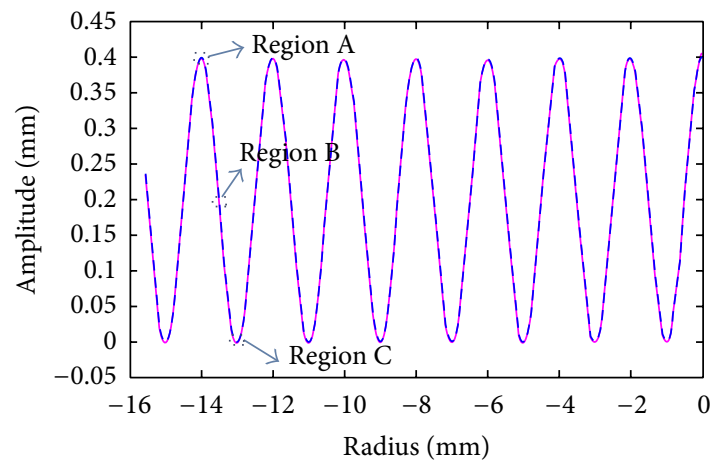

_ Measuring curve

- - - Theoretical curve

(b)

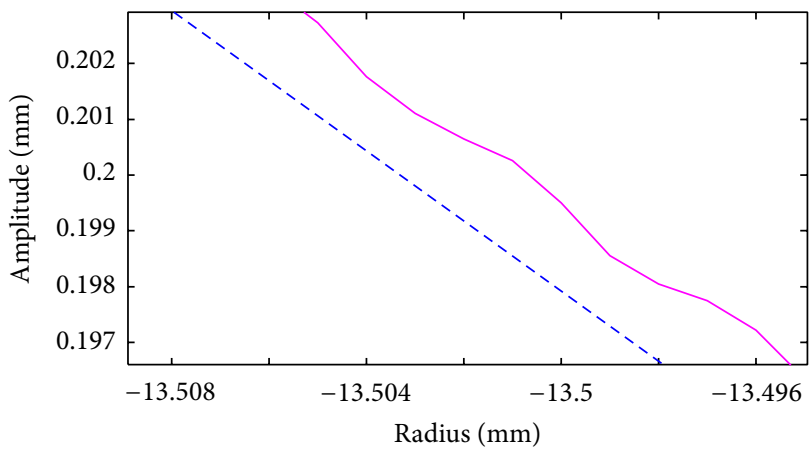

- Measuring curve

- - - Theoretical curve

(d)

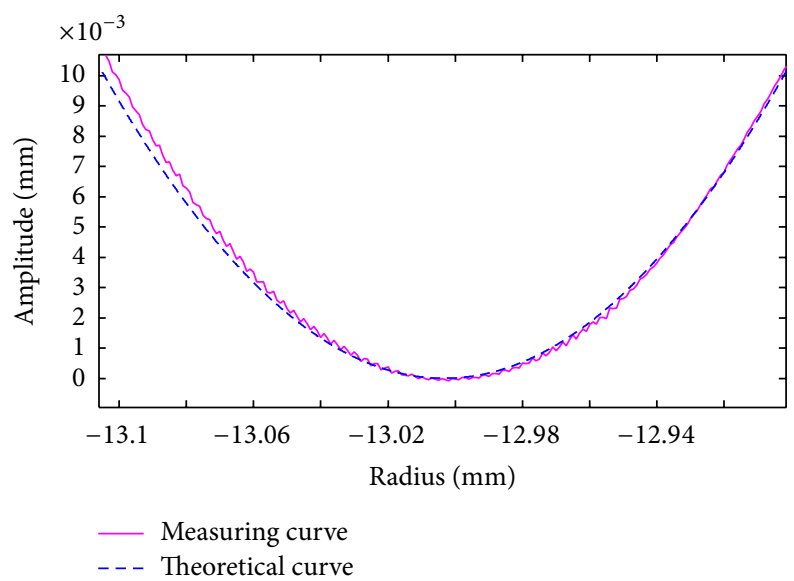

(e)

Figure 10: Analysis figures of the $1 \mu \mathrm{m}$ measured curve. (a) Original measured curve, (b) half of the original measured curve, (c) enlarged graph of "Region A," (d) enlarged graph of "Region B," and (e) enlarged graph of "Region C." 


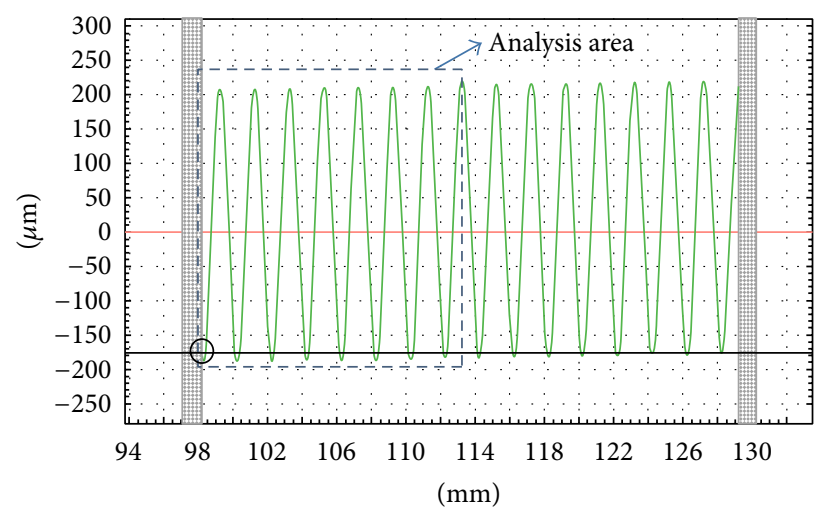

(a)

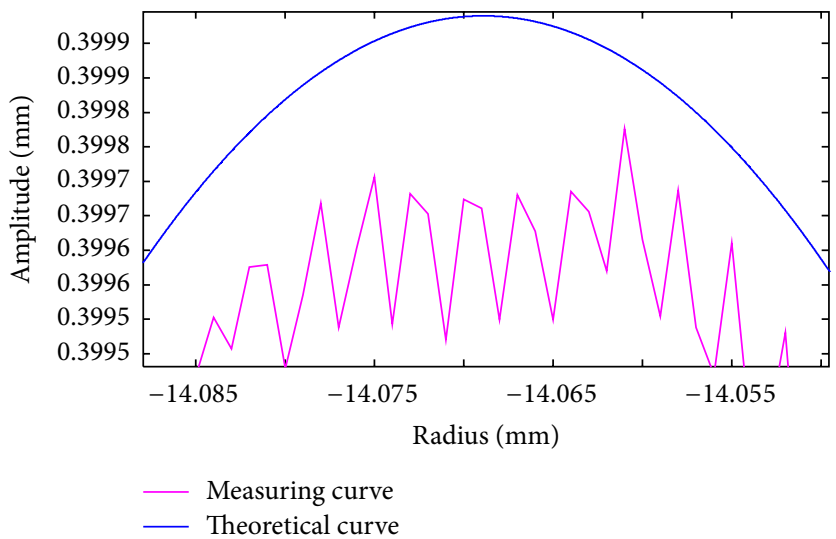

(c)

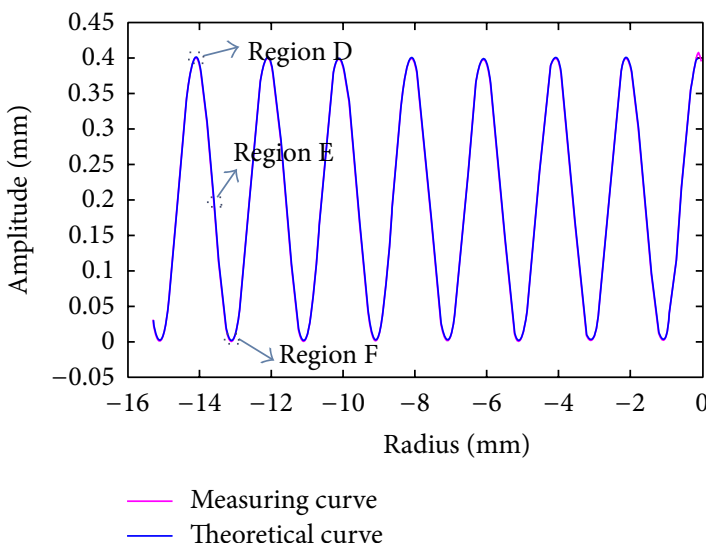

(b)

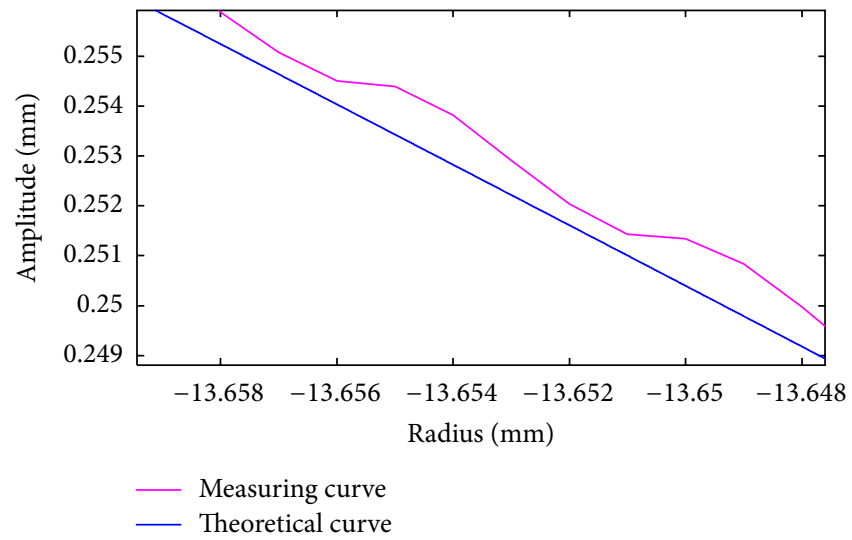

(d)

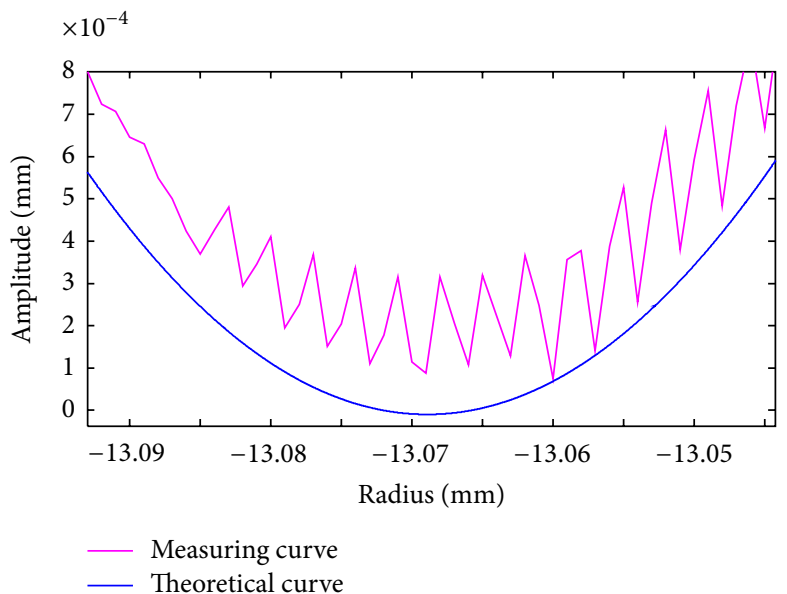

(e)

Figure 11: Analysis figures of the $0.1 \mu \mathrm{m}$ measured curve. (a) Original measured curve, (b) half of the original measured curve, (c) enlarged graph of "Region D," (d) enlarged graph of "Region E," and (e) enlarged graph of "Region F." 


\section{Conflict of Interests}

The authors declare that there is no conflict of interests regarding the publication of this paper.

\section{Acknowledgments}

This work is supported by the National Key Basic Research and Development Program (973 Program) of China (Grant no. 2011CB706702), Natural Science Foundation of China (Grants nos. 51305161 and 51135006), and Jilin province science and technology development plan item (Grant no. 20130101042JC).

\section{References}

[1] X. D. Zhang, F. Z. Fang, Q. Q. Wu, X. L. Liu, and H. M. Gao, "Coordinate transformation machining of off-axis aspheric mirrors," The International Journal of Advanced Manufacturing Technology, vol. 67, no. 9-12, pp. 2217-2224, 2013.

[2] http://www.tydexoptics.com/products1/spectroscopy/oap_mirrors/.

[3] Z. Q. Yin, Y. F. Dai, S. Y. Li, C. L. Guan, and G. P. Tie, "Fabrication of off-axis aspheric surfaces using a slow tool servo," International Journal of Machine Tools and Manufacture, vol. 51, no. 5, pp. 404-410, 2011.

[4] J. Yan, Z. Zhang, T. Kuriyagawa, and H. Gonda, "Fabricating micro-structured surface by using single-crystalline diamond endmill," International Journal of Advanced Manufacturing Technology, vol. 51, no. 9-12, pp. 957-964, 2010.

[5] S. J. Zhang, F. Z. Fang, Z. W. Xu, and X. T. Hu, "Controlled morphology of microtools shaped using focused ion beam milling technique," Journal of Vacuum Science \& Technology B: Microelectronics and Nanometer Structures, vol. 27, no. 3, pp. 1304-1309, 2009.

[6] W. H. Yang and Y. S. Tarng, "Design optimization of cutting parameters for turning operations based on the Taguchi method," Journal of Materials Processing Technology, vol. 84, no. 1-3, pp. 122-129, 1998 .

[7] R. Q. Sardiñas, M. R. Santana, and E. A. Brindis, "Genetic algorithm-based multi-objective optimization of cutting parameters in turning processes," Engineering Applications of Artificial Intelligence, vol. 19, no. 2, pp. 127-133, 2006.

[8] M. Tolouei-Rad and I. M. Bidhendi, "On the optimization of machining parameters for milling operations," International Journal of Machine Tools and Manufacture, vol. 37, no. 1, pp. 1-16, 1997.

[9] R. M. Patrikar, "Modeling and simulation of surface roughness," Applied Surface Science, vol. 228, no. 1-4, pp. 213-220, 2004.

[10] P. G. Benardos and G.-C. Vosniakos, "Predicting surface roughness in machining: a review," International Journal of Machine Tools and Manufacture, vol. 43, no. 8, pp. 833-844, 2003.

[11] Y. Tohme, R. Murray, and E. Allaire, "Principles and applications of the slow slide servo," Moore Nanotechnology Systems White Paper, 2005.

[12] W. Gao, T. Araki, S. Kiyono, Y. Okazaki, and M. Yamanaka, "Precision nano-fabrication and evaluation of a large area sinusoidal grid surface for a surface encoder," Precision Engineering, vol. 27, no. 3, pp. 289-298, 2003.
[13] T. A. Dow, M. H. Miller, and P. J. Falter, "Application of a fast tool servo for diamond turning of nonrotationally symmetric surfaces," Precision Engineering, vol. 13, no. 4, pp. 243-250, 1991.

[14] A. Y. Yi and L. Li, "Design and fabrication of a microlens array by use of a slow tool servo," Optics Letters, vol. 30, no. 13, pp. 1707-1709, 2005.

[15] L. Li, A. Y. Yi, C. Huang, D. A. Grewell, A. Benatar, and Y. Chen, "Fabrication of diffractive optics by use of slow tool servo diamond turning process," Optical Engineering, vol. 45, no. 11, Article ID 113401, 2006.

[16] S. To, T. C. Kwok, C. F. Cheung, and W. B. Lee, "Study of ultraprecision diamond turning of a microlens array with a fast tool servo system," in 2nd International Symposium on Advanced Optical Manufacturing and Testing Technologies: Advanced Optical Manufacturing Technologies, vol. 6149 of Proceeding of SPIE, November 2005. 


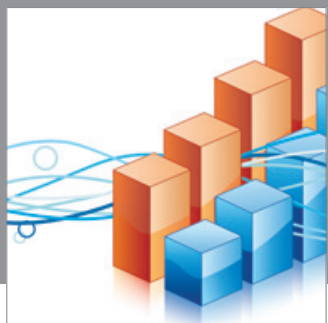

Advances in

Operations Research

mansans

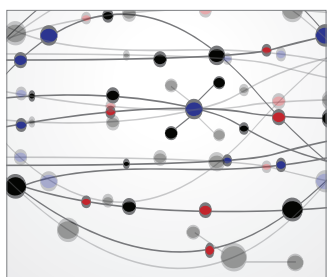

The Scientific World Journal
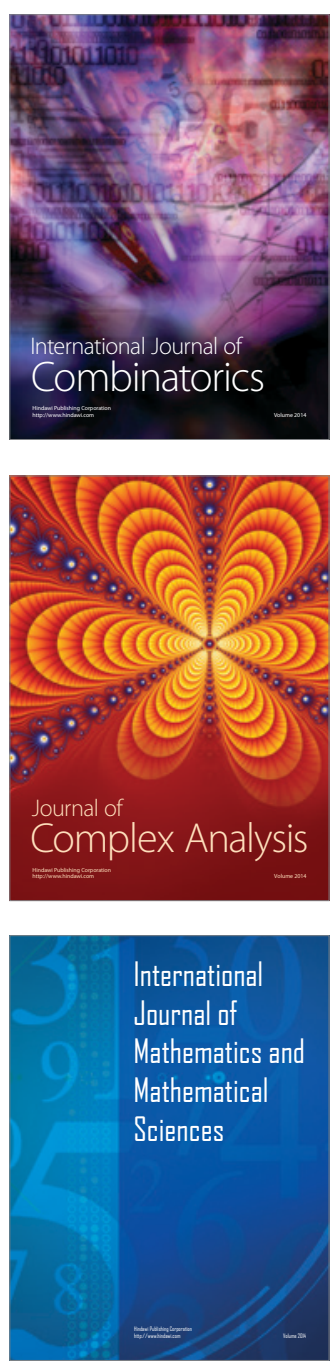
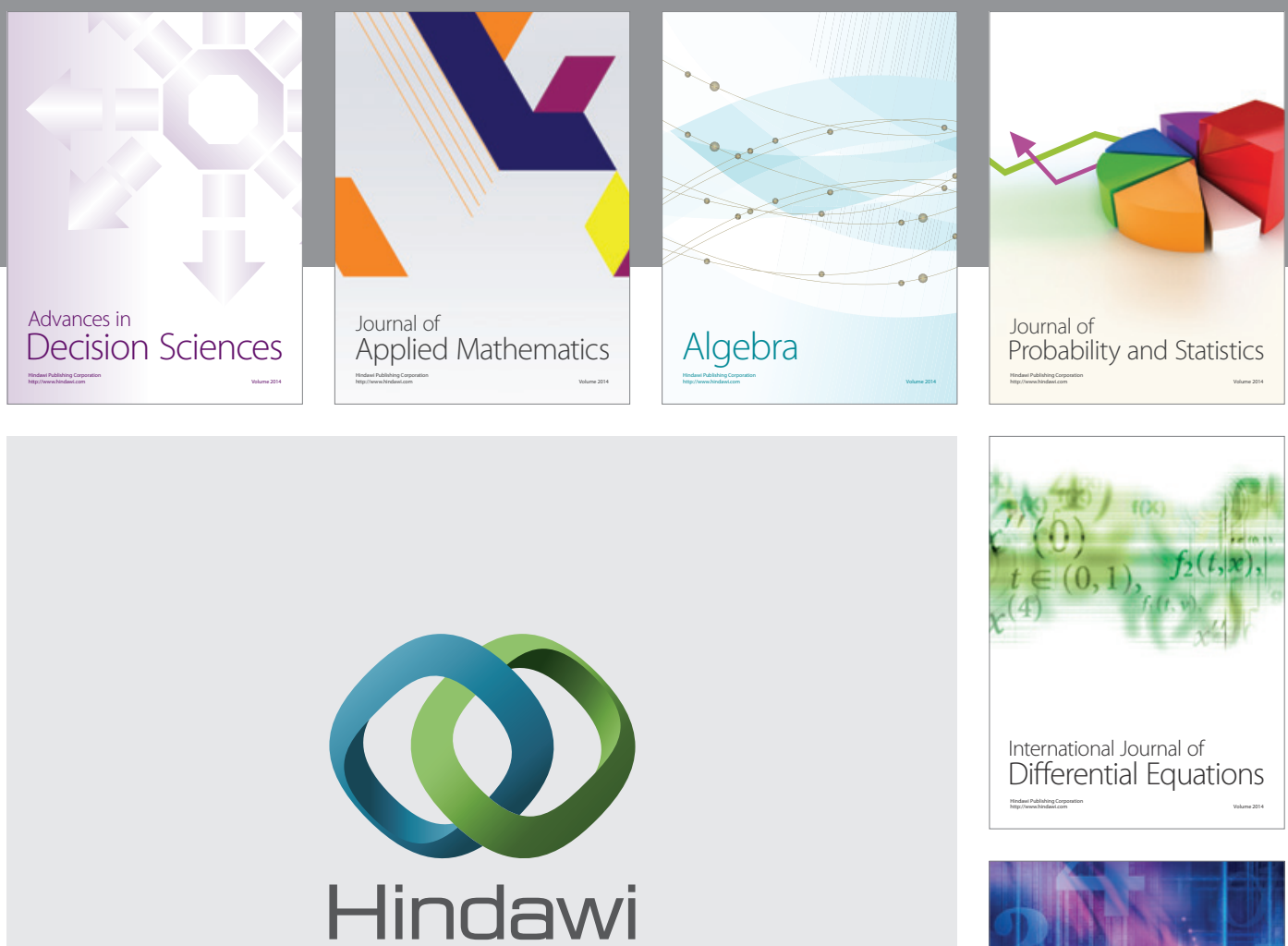

Submit your manuscripts at http://www.hindawi.com
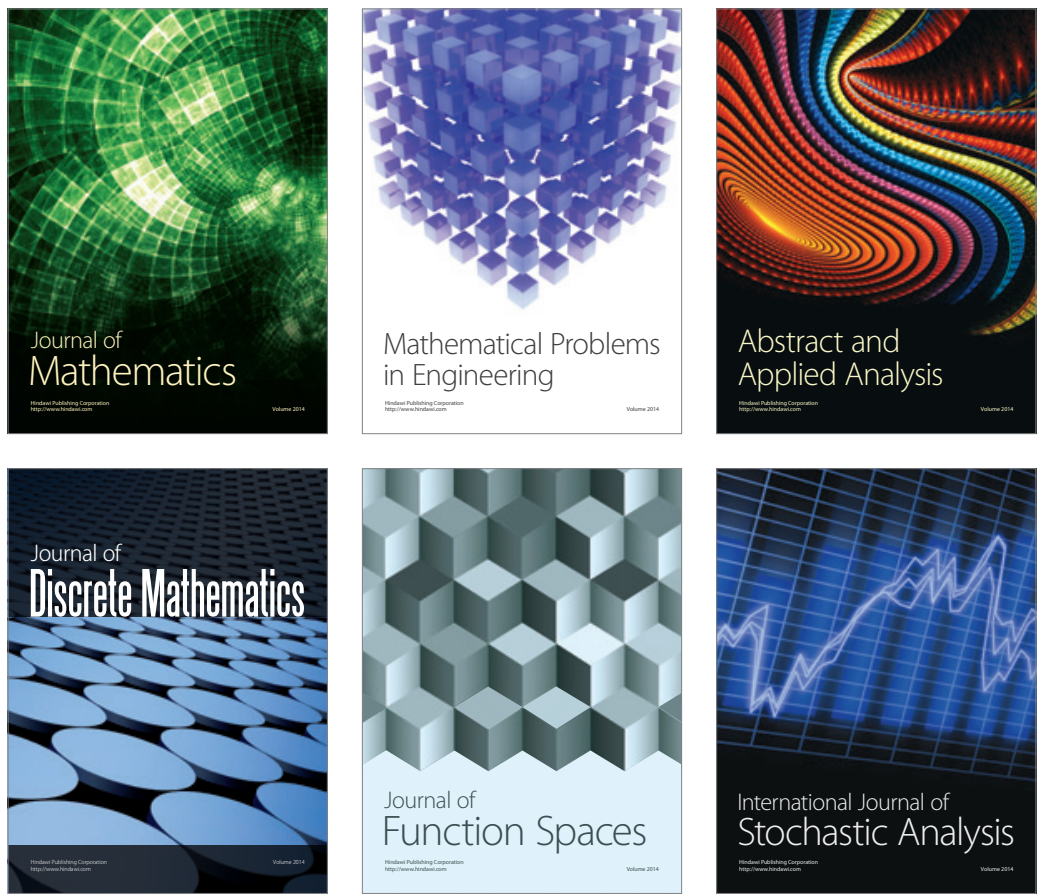

Journal of

Function Spaces

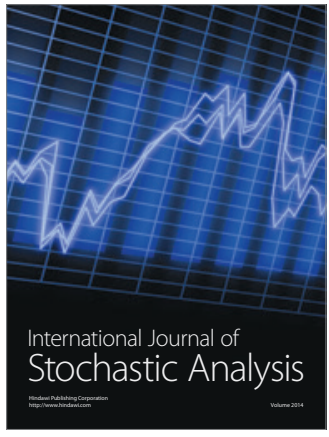

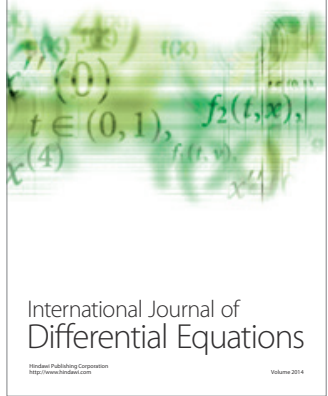
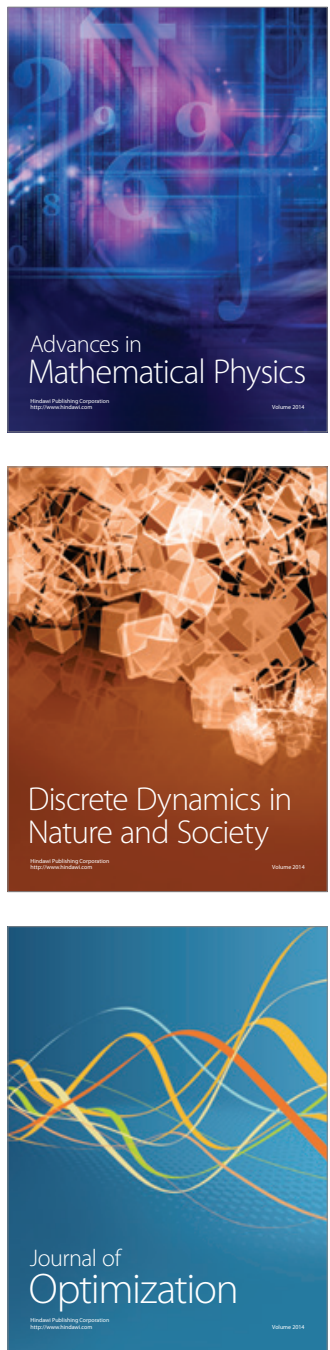